\title{
MULTI-MEV ION BEAMS FROM TERAWATT LASER THIN-FOIL INTERACTIONS
}

\author{
K. Flippo*, A. Maksimchuk, S. Banerjee, V. Wong, G. Mourou, D. Umstadter, Center for Ultrafast \\ Optical Science, University of Michigan, Ann Arbor, MI 48109-2099, USA \\ K. Nemoto, Central Research Institute of Electrical Power Industry, 2-11-1, Iwado-kita, Komae-shi, \\ Tokyo, 201-8511 Japan \\ V. Yu. Bychenkov, P.N. Lebedev Physics Institue, Russian Academy of Science, 117924 Moscow, \\ Russia
}

\begin{abstract}
We have conducted investigations of a collimated beam of fast protons, produced by a 10TW laser with frequencies of either $\omega_{0}$ (corresponding to 1.053 micron light) or $2 \omega_{0}$ (corresponding to $532 \mathrm{~nm}$ light) focused to an intensity of more the $3 \times 10^{19} \mathrm{~W} / \mathrm{cm}^{2}$ onto the surface of a thin-film target. Energies as high as $10 \mathrm{MeV}$ and total number of $10^{\circ}$, confined in a cone angle of $40^{\circ} \pm 10^{\circ}$ have been observed. The protons, which originate form impurities on the front side of the target and exit out the backside in a direction normal to the target surface. Acceleration field gradients of $\sim 10 \mathrm{GeV} / \mathrm{cm}$ have been inferred. The maximum proton energy for $2 \omega_{0}$ can be explained by the charge-separation electrostatic-field acceleration due to "vacuum heating." In another set of experiments when a deuterated polystyrene layer was deposited on a surface of a Mylar film and a ${ }^{10} \mathrm{~B}$ sample was placed behind the target, we observed the production of $\sim 10^{5}$ atoms of positron active isotope ${ }^{11} \mathrm{C}$ from the nuclear fusion reaction ${ }^{10} \mathrm{~B}(\mathrm{~d}, \mathrm{n}){ }^{11} \mathrm{C}$. No activation was detected when only the proton beam was used. We also discuss the use of ions from these table-top sources for medical applications such as cancer radiotherapy and fundamental studies in radio-biology.
\end{abstract}

\section{INTRODUCTION}

Recently the advent of ultra-intense lasers using the Chirped Pulse Amplification technique has made possible the study of processes occurring at intensities in excess of $10^{18} \mathrm{~W} / \mathrm{cm}^{2}$. At these very high intensities the electrons can acquire energy, which can be equal to or larger then the electron's rest mass, in a half laser cycle. Charge separation of these hot electrons can then give rise to large electrostatic fields, which can accelerate the surrounding ions to $\mathrm{MeV}$ energies in very short distances.

Interest in compact high-intensity subpicosecond-laserdriven ion accelerators for applications including neutron sources, short-lived isotope nuclear physics, medical isotope production and cancer therapies has grown in recent years. Experiments generating high energy ions have been accomplished using gas jets[1,2], clusters[3,4] and solid targets[5-11].

\footnotetext{
*kflippo@umich.edu
}

Ion acceleration depends on the generation of high energy electrons, which, via charge separation, create the very strong electrostatic fields needed for the production of high energy ions. Two well know mechanisms for charge separation are the thermal expansion of the laserdriven plasma, and the ponderomotive electron expulsion.

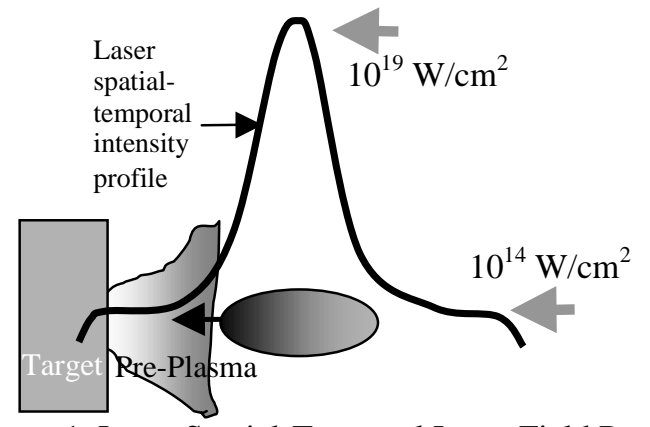

Figure 1. Laser Spatial-Temporal Laser Field Profile

\section{ION PRODUCITON AND CONTRAST}

We have observed the production of protons using several different target materials, including carbon, molybdenum, lead, aluminum, and Mylar at different laser wavelengths, 1.053 microns and 532 nanometers, which have inherently different laser contrasts. This laser parameter affects the ion energy scaling significantly. The laser contrast is a measure of the intensity ratio of the main pulse to the intensity of the pedestal the main pulse rides upon. The pedestal is a consequence of the way the laser is produced. The lasing medium has a characteristic decay time during which this medium can emit amplified spontaneous emission (ASE), coherent radiation which can precede the main pluse by nanoseconds. The ASE is amplified along with the main pulse along the chain. Typical contrast for a Nd: Glass system is on the order of $10^{-5}: 1$, such that a laser with the intensity of $10^{19} \mathrm{~W} / \mathrm{cm}^{2}$ would have a "pre-pulse" on the order of $10^{14} \mathrm{~W} / \mathrm{cm}^{2}$. In some cases this "pre-pulse" may have enough energy to create a substantial plasma before the main pulse arrives, leading to an underdense laser-plasma interaction and not the desired solid-laser interface. We have used second harmonic generation to double the frequency of our laser, and improve the contrast to an estimated $10^{-7}: 1$. 


\subsection{Experiments at High Contrast, $2 \omega_{0}$}

In a previous experiment [9] the frequency doubled laser light $(532 \mathrm{~nm})$ was shone on films of $1.8 \mu \mathrm{m}$ Aluminum at $0^{\circ}$ and $45^{\circ}$ incidence, focused to an intensity of $3 \times 10^{18} \mathrm{~W} / \mathrm{cm}^{2}$. CR-39 was used to record the protons (sensitive to $100 \mathrm{keV} /$ nucleon), and their energies were measured using thin foils to attenuate their energy, as well, a simple dipole spectrometer was employed.

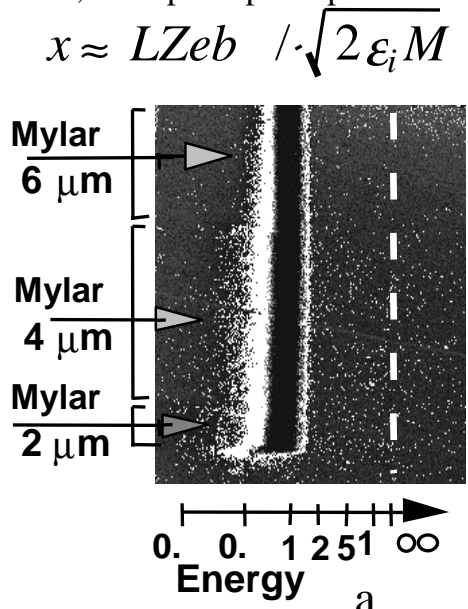

a

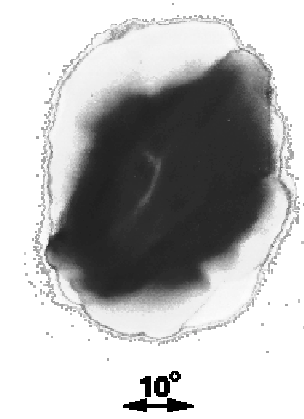

b

Figure 2: (a) Proton spectra from a dipole magnet spectrometer with steps of Mylar filters of 2,4,6 microns covering the CR-39 detector. (b) Image of beam above 1.2 $\mathrm{MeV}$, showing a 40-degree divergence.

Figure 2a shows the proton spectrum from a shot at $2 \omega_{0}$ and $3 \times 10^{18} \mathrm{~W} / \mathrm{cm}^{2}$. The maximum proton energy is around $2 \mathrm{MeV}$ with a total of around $10^{10}$ protons, with $10^{9}$ above $1 \mathrm{MeV}$. The maximum proton energy scaled as the laser intensity, I, to the 0.4 power. $2 \mathrm{~b}$ shows the structure and divergence of the beam, which is about 40 degrees.

\subsection{Experiments at Low Contrast, $\omega_{0}$}

The laser at its fundamental frequency (1.053 microns) has a contrast of $10^{-5}: 1$, which can at very small focal spots/ high intensities lead to the formation of a preplasma at the laser-solid interface. This pre-plasma scale length effects the production of hot electrons and thus the efficiency of proton acceleration.

With an $\mathrm{f} / 3.3$ paraboloid the laser could be focused to a diameter spot of between 10 and 12 microns FWHM, yielding a maximum intensity on target of $5 \times 10^{18} \mathrm{~W} / \mathrm{cm}^{2}$. From this interaction we have observed protons of up to $10 \mathrm{MeV}$, with a nearly linear scaling with respect to the laser intensity, I.

Using an $\mathrm{f} / 1.5$ parabola a laser spot size a little over 4 microns FWHM was achieved, yielding a maximum intensity of $3 \times 10^{19} \mathrm{~W} / \mathrm{cm}^{2}$ on target. Under these conditions our prepulse intensity has increased by a factor of six, the laser spot area decreased by a factor of six, and we have observed a maximum proton energy of $5 \mathrm{MeV}$ with a scaling on the order of $\mathrm{I}^{5}$. This decrease in proton energy by a factor of 2 may be due to the formation of a higher density pre-plasma, which may lead to the inhibition of hot electron production and charge separation by the very strong magnetic fields induced in the target along with possible space charge effects from the decrease scale.

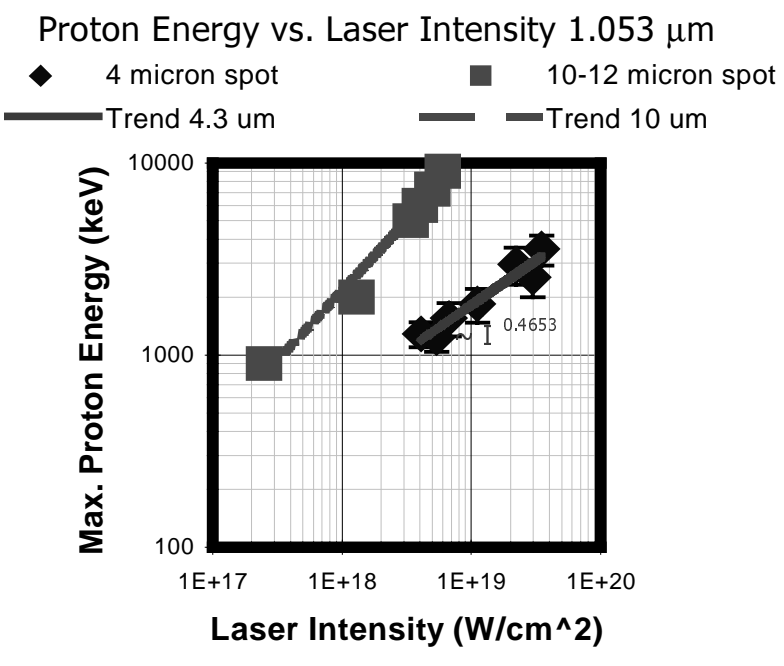

Figure 3: Maximum proton energy versus laser intensity for the laser fundamental frequency reveals a difference in maximum proton energy and scaling for different laser spot sizes.

We believe we have observed the effect of these intense fields by examining the emitted proton beam incident on to CR-39, $3 \mathrm{~cm}$ from the target's non-laser-irradiated (back) surface, figure 4 . The beam from the high intensity 4-micron spot stands in strong contrast to beam from the larger, lower intensity, 10 micron spot size, which is relatively uniform and has a weak internal structure similar to the $2 \omega_{0}$ case shown in figure $2 \mathrm{~b}$. At the high intensities of the 4-micron focus a strong internal structure of the proton beam has developed, which tends to be symmetric in nature, and has a smaller divergence then the high contrast, about 30 degrees.

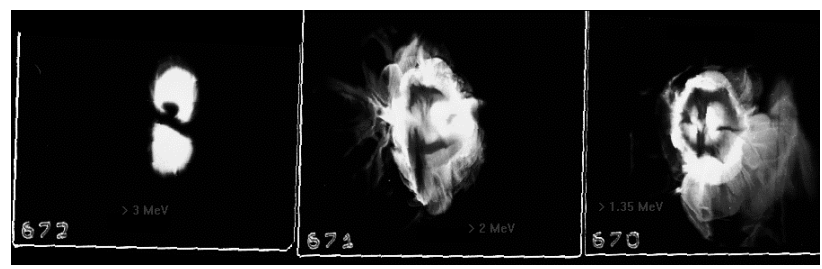

Figure 4: Images of the emitted proton beam at different energies using CR-39. Image 672, above $3 \mathrm{MeV}$; 671 above $3 \mathrm{MeV}$; and 670 above $1.35 \mathrm{MeV}$

\subsection{Protons from the laser irradiated surface}

There has been some controversy over whether the protons from thin film targets are being accelerated from the front surface $[7,9]$ or the back surface $[8,11]$. To 
determine the acceleration distance of the protons we performed experiments on various target thicknesses.

This had been done previously with Aluminum [9], and now we have done it with Mylar, figure 5. The trends are very similar, both $\mathrm{Al}$ and Mylar exhibit an increase in proton energy until a thickness of between 10 and 13 microns then begin to decline. If the energy loss $(\mathrm{dE} / \mathrm{dx})$ of the protons traveling through the target is taken into account (solid lines) we see a saturation effect. This can be taken as evidence that the acceleration distance is very short (microns) and that the protons are indeed being accelerated from the laser irradiated (front) surface.

\section{Proton Energy vs. Target Thickness}

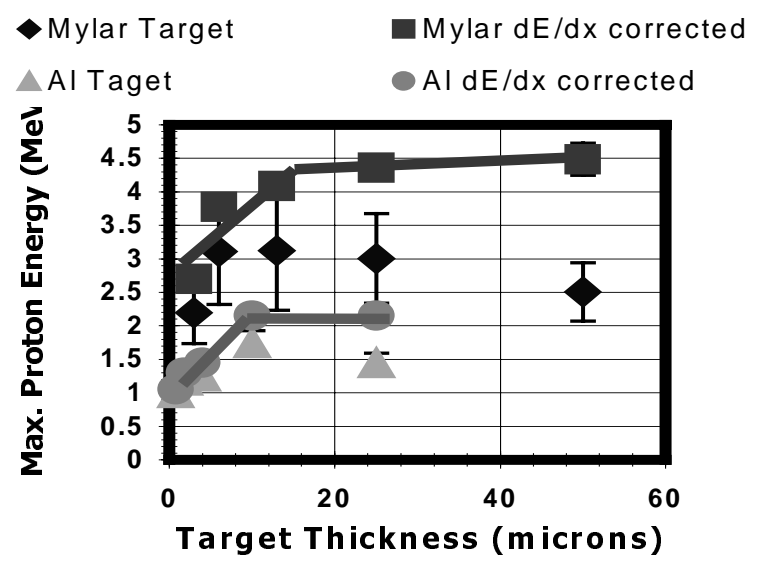

Figure 5: Maximum proton energy varies with target thickness, for $\mathrm{Al}$ and Mylar. If the target thickness is accounted for (assuming front side acceleration) then a saturation effect is observed.

To corroborate this data we performed [10] an experiment where we activated a sample of ${ }^{10} \mathrm{~B}$ via the nuclear reaction ${ }^{10} \mathrm{~B}(\mathrm{~d}, \mathrm{n}){ }^{11} \mathrm{C}$, using deuterons accelerated from the front surface of our targets. No activation was observed when the back of the target was deuterated or when no deuterons were present on either surface of the Mylar target.

\section{CONCLUSIONS AND FUTURE WORK}

In conclusion we have shown that the maximum proton energy scales to the laser intensity between $\mathrm{I}^{3}$ and $\mathrm{I}^{4}$ for high contrast [9], and from approximately $\mathrm{I}$ to $\mathrm{I}^{5}$ for low contrast. Also for the low contrast case the beam divergence decrease to 30-degrees down from 40-degrees for the high contrast case. We have also observed a decrease in the maximum proton energy when a smaller focal spot, with higher intensity was used. Reasons for this may be related to the pre-plasma scale length and the formation of intense magnetic fields inside the target, which have been inferred from images of the emitted proton beam.

We have also previously demonstrated the use of the electron beam from a gas target to do pulse radiolysis of water [12]. In the future the proton beam could be similarly used as an ultrafast probe of solids, or used to produce an ultrashort bust of neutrons for the same purpose.

We have also already shown the real capability of isotope production from a laser accelerator system for either medical applications or short-lived isotope studies [10].

Future work involves the use of the proton beam to test its ability to treat cancerous tissues, such as melanoma or cancers of the eye. A fast bunch of protons may have the advantage of causing less collateral damage to healthy tissues around the cancer then that of a traditional RF bunched beam.

This work is supported by the Division of High Energy Physics, Office of Energy Research, U.S. Department of Energy, award DE-FG02-98ER41071, with lasers supported by the National Science Foundation Center for Ultrafast Optical Science

\section{REFERENCES}

[1] G. S. Sarkisov et al., JETP Lett. 66, 828 (1997); G. S. Sarkisov et al., Phys. Rev. E 59, 7042 (1999).

[2] K. Krushelnick et al. "Multi-MeV ion production from high-intensity laser interactions with underdense plasmas," Phys. Rev. Lett. 83, 737 (1999).

[3] T. Ditmire et al.," Nature (London) 386, 54 (1997).

[4] M. Lezius et al., Phys. Rev. Lett. 80, 261 (1998).

[5] A. P. Fews et al., "Plasma ion emissions from gigh intensity picosecond laser pulse interactions with solid targets," Phys. Rev. Lett, 73, 1801 (1994); F. N. Beg et al., Phys. Plasmas 4, 447 (1997).

[6] P. E. Young et al., "Fast ion production by laser filamentation in laser-produced plasmas," Phys. Rev. Lett. 76, 3128 (1996).

[7] E.L. Clark et al., "Measurements of energetic proton transport through magnetized plasma from intense laser interactions with solids," Phys. Rev. Lett. 84, 670 (2000); E.L Clark et al., "Measurements of energetic proton transport through magnetized plasma from intense laser interactions with solids,' Phys. Rev. Lett. 85, 1654 (2000) [8] R.A. Snavely et al., "Intense high-energy protn beam from petawatt-laser irradiation of solids," Phys. Rev. Lett. 85, 2945 (2000)

[9]A. Maksimchuck, S. Gu, K. Flippo, et al., "Forward ion acceleration in thein films driven by a high-intensity laser," Phys. Rev. Lett., 84, 4108 (2000)

[10]K. Nemoto et al., "Laser-triggered ion acceleration and table top isotope production," Appl. Phys. Lett., 78, 5955 (2000)

[11] T.E. Cowan et al., "Photonuclear fission from high energy electrons from ultraintense laser-solid interactions," Phys. Rev. Lett., 84, 903 (2000) [12] N. Saleh et al, "Pulse radiolysis of liquid water using picosecond electron pulses produced by a table-top terawatt laser system," Rev. Sci. Inst., 71, 2305-8 (2000) 